\title{
Firing Properties of Hippocampal Neurons in a Visually Symmetrical Environment: Contributions of Multiple Sensory Cues and Mnemonic Processes
}

\author{
Patricia E. Sharp, ${ }^{1}$ John L. Kubie, ${ }^{2}$ and Robert U. Muller ${ }^{1}$ \\ Departments of 'Physiology and ${ }^{2}$ Anatomy and Cell Biology, SUNY Health Sciences Center at Brooklyn, Brooklyn, \\ New York 11203
}

The location-specific firing of hippocampal place cells can easily be brought under the control of experimenter-defined cues. Nevertheless, there is evidence that these firing fields are not determined just by immediate sensory input, but also by earlier states of the nervous system (O'Keefe and Speakman, 1987). Here, we report further on the roles of multiple visual cues and mnemonic processes in determining the firing of place cells.

Rats were trained to chase food pellets in a cylinder with homogeneous gray walls and 1 white cue card. After a cell's field was recorded in this "standard" condition, probe sessions were conducted in which a second card was placed $180^{\circ}$ away from the first. This configuration created a diametrically symmetrical environment in which pairs of locations $180^{\circ}$ apart were identical with respect to views of the wall and cards. If place cells are strongly controlled by these immediately available views, firing in the 2-card configuration should be diametrically symmetrical. Alternatively, because the rat moves freely in the cylinder, information is available that pairs of visually identical places are not truly the same. If some mnemonic process stores and updates information about the rat's paths during the session, it is possible that the firing pattern will be different in 2 such places, especially because the original training was conducted in the 1-card, asymmetrical environment.

Thirteen of 18 cells had a single, asymmetric firing pattern after the second card was introduced; the field was the same size and shape as in the 1-card configuration and in the same spatial relation to 1 of the 2 cards. The field position during 2 -card sessions could be rotated $180^{\circ}$ by starting the rat by one card or the other. In further probe sessions in which the cue cards, entry location, and background cues were, in various combinations, rotated in relation to each other, these cells always showed a single field, similar in size and shape to that in the standard, and in the same relationship as in the standard to as many cues as possible.

The remaining 5 cells showed complex changes over repeated 2-card sessions, and 3 of these showed paired fields,

\footnotetext{
Received Dec. 12, 1989; revised May 7, 1990; accepted May 8, 1990.

This work was supported by NIH Grant NS 20686 to J.L.K. and R.U.M. and by NINCDS Grant NS 08408 to P.E.S. We thank Dr. James B. Ranck for many useful discussions.

Correspondence should be addressed to Dr. Patricia E. Sharp, Department of Psychology, P.O. Box 11 A, Yale Station, Yale University, New Haven, CT 06520.

Copyright (C) 1990 Society for Neuroscience $0270-6474 / 90 / 093093-13 \$ 03.00 / 0$
}

$180^{\circ}$ apart for at least some of the sessions. In one case, the second field disappeared with repeated exposures to 2 cards; in another, the second field persisted when only 1 card was used. We conclude that place cells are influenced both by the immediate sensory configuration and by internal neural states related to earlier experience in the environment.

Complex-spike cells in the hippocampus show location-specific firing as an animal moves about an environment (O'Keefe and Dostrovsky, 1971; O'Keefe, 1976). These units, which are called "place cells," fire rapidly only when the animal is in a restricted part of its environment; the restricted regions of intense firing are called "firing fields." The location of firing fields can easily be brought under the control of experimenter-defined cues. For instance, rotation of the cues results in equal rotation of placecell firing fields (O'Keefe and Conway, 1978; Muller and Kubie, 1987).

Previous work on the manner in which cues determine spatial firing patterns used conditions in which some or all of the controlled cues were removed. O'Keefe and Conway (1978) showed that, when any subset of the experimenter-defined cues is removed, fields in many cases are unaffected, regardless of which particular cues are removed. Additional studies have shown that fields can maintain their position when all visual cues are removed, provided that the animal is first allowed to orient to the situation. Specifically, if an animal is placed into a familiar environment and the room lights are subsequently extinguished or experimenter-defined cues are removed, place cells retain their original firing field (O'Keefe, 1976; Jones-Leonard et al., 1985; O'Keefe and Speakman, 1987; McNaughton et al., 1989; Quirk et al., 1990). However, if the animal is introduced into the environment in the dark or after the cues have been removed, then the field may show a completely different pattern. Therefore, the ability of the cell to maintain its field in the dark depends on the rat's original exposure to the environment when the cues are still available.

Two general types of factors can be postulated to account for the phenomenon of location-specific firing. The first type, which we call "sensory," refers to the set of sensory cues immediately available to the animal from any location in the environment. Because different locations offer different views, this type of information could clearly support place-cell firing (Zipser, 1985; McNaughton, 1989). The results from the cue-rotation experiments described above are consistent with an exclusively sensory model. The results from cue-elimination experiments sug- 

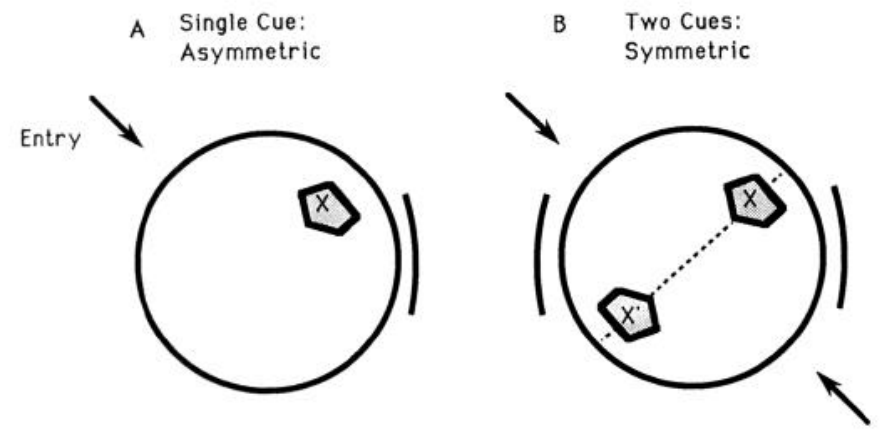

Figure 1. Schematic representation of overhead views of cylinder used during 1-card $(A)$ and 2-card $(B)$ recording sessions. The positions of the cards (which are actually mounted on the inside wall of the cylinder) are indicated by the arcs located at 3:00 and 9:00, and the entry location is indicated by an arrow. Note that, for the 2-card sessions, there were 2 possible entry locations, only 1 of which was used for any one session. In the 2 -card configuration, the viewpoints available from any 2 locations that are $180^{\circ}$ apart and equidistant from the center (e.g., $X$ and $\left.X^{\prime}\right)$ are indistinguishable.

gest, however, that the model is not a complete explanation. The second type of factor, which we call "trace cues," refers to cues that are not currently available to the rat; if such cues influence place-cell firing, their effects must be mediated in part by memory mechanisms. Trace cues can include past environmental events, as well as integrated path signals based on past motor behavior. It is useful to refer to stored information about a particular environment as a mnemonic representation of that environment.

Results from the cue-elimination studies have led to the idea that hippocampal activity must be based in part on stored representations of the original training environment. It has been hypothesized that stored representations can be used, along with information about the rat's current motor behavior, to generate appropriate patterns of location-specific firing as an animal moves about an environment after some or all the controlling stimuli have been removed (McNaughton, 1989).

The present study was designed to investigate further the manner in which sensory factors and mnemonic factors determine a cell's firing patterns. In this study, however, instead of removing salient cues, we duplicated them. Rather than asking whether firing fields can be generated in a reduced version of the original training situation, we asked what firing patterns will be produced when the system is presented with more than 1 location within an environment that is similar to a given location in the original.

Animals were trained to perform a pellet-chasing task in a cylindrical apparatus with a single white cue card on the wall (see Fig. 1A). In previous studies, it has been shown that cue cards of this sort control the angular orientation of firing fields (Bostock et al., 1986; Muller and Kubie, 1987). The cylinder with a single card is called the "standard" configuration; it is the same as the configuration used in previous studies and was used during behavioral training in this study. The main manipulation used here was to add a second, identical white card $180^{\circ}$ from the first (Fig. 1B). We call this the "symmetric environment" because it creates an apparatus with diametric symmetry.

In the symmetric environment, the view from any location is always identical to the view the animal sees from a second location across the diameter and equally distant from the cylinder center. The pair of points $\mathrm{X}$ and $\mathrm{X}^{\prime}$ in Figure $1 B$ is an example of a pair of ambiguous locations. In this case, locations $\mathrm{X}$ and $\mathrm{X}^{\prime}$ in Figure $1 B$ are associated with stimulus configurations similar to location $\mathrm{X}$ in the standard situation (Fig. 1A).

There are 3 extreme outcomes that are compatible with previous studies. If sensory factors weigh most heavily, it might be expected that there would be a doubling of the original field, because a salient cue has been duplicated. Alternatively, if trace factors are important and the animal's past representation of the asymmetrical environment is a strong determinant, the asymmetrical field might be retained. Therefore, firing in 1 of the 2 visually similar places might be suppressed. Finally, the 2-cue environment might be sufficiently different from the standard situation so that a completely new field will result.

Because previous cue-removal studies suggested that initial exposure to an environment is important for setting up the hippocampal representation, it was reasoned that the rat's entry location into the cylinder might be important. The arrow in Figure $1 A$ indicates the location at which the animal was placed into the cylinder on standard sessions. During 2-card sessions, there were 2 possible entry points, with each 1 bearing the same relationship as in the standard to one of the cards.

The majority of cells showed an asymmetric firing pattern in the 2-cue situation. The field was similar in size and shape to that in the standard situation and had the same spatial relationship to entry angle and to 1 of the 2 cue cards that it had in the standard situation. Therefore, these cells did not fire in each of the 2 places that were similar to their fields in the presence of 1 card. Instead, they fired in only 1 of the 2 symmetric places during a given session, such that the field location was compatible with entry angle.

Further probes in which the cards, entry location, and background cues were placed in nonstandard relationships to each other showed that the cells have a strong tendency to show a firing pattern similar in size and shape to that in the standard and also in the location in register with as many of the cues as possible.

These findings are compatible with those from previous studies because they show that the firing of place cells is controlled by multiple cues. They go a step further, however, in illustrating the manner in which multiple cues are utilized. From previous studies showing the existence of place fields under impoverished conditions, one outcome that might have been expected is that, when a salient stimulus is duplicated or stimuli are rotated in relation to each other, multiple fields would be exhibited so there would be 1 field for each place that was visually similar to the location of the cell's original field. This is not the case, however; we observed, instead, that most cells within the hippocampus choose a single firing pattern closest to that in the original learning situation.

A second important finding is that a minority of cells do show doubling of firing fields. This demonstrates that location-specific sensory factors also play an important role.

\section{Materials and Methods}

The basic behavioral, physiological, and data-collection methods used here have been presented previously (Muller et al., 1987) but will be described briefly below.

Experimental subjects. The experimental subjects were 12 female, hooded, Long-Evans rats, obtained from Blue Spruce Farms and weighing $250 \mathrm{gm}$ at the time of shipping.

Apparatus. The recording chamber was a $76-\mathrm{cm}$-diameter, $51-\mathrm{cm}-$ 
Table 1

Results of rotational correlation analysis for cells in Category 1

\begin{tabular}{|c|c|c|c|c|c|}
\hline Session Name & Session description & Diagram & $N$ & $\begin{array}{c}\text { Angle of } \\
\operatorname{Max} R\end{array}$ & $\operatorname{Max} R$ \\
\hline Standard & $\begin{array}{l}\text { Single card, standard } \\
\text { (A) entry }\end{array}$ & & 8 & $2.7^{\circ} \quad(3.2)$ & .67 \\
\hline 2-card-A & $\begin{array}{l}\text { 2-Card with standard } \\
\text { (A) entry }\end{array}$ & & 12 & $-2.3^{\circ}(2.3)$ & .70 \\
\hline $2-\operatorname{card}-\mathrm{B}$ & $\begin{array}{l}\text { 2-Card with rotated }(B) \\
\text { entry }\end{array}$ & & 12 & $182.5^{\circ}(3.5)$ & .60 \\
\hline 2 -card $(+)$ nudge & $\begin{array}{l}\text { 2-Card -- } 30^{\circ} \\
\text { clockwise card rotation } \\
\text { and rotated }(B) \text { entry }\end{array}$ & & 4 & $158.3^{\circ}(5.5)$ & .65 \\
\hline 2-card (-) nudge & $\begin{array}{l}\text { 2-Card }-30^{\circ} \\
\text { counterclockwise card } \\
\text { rotation and rotated }(B) \\
\text { entry }\end{array}$ & & 4 & $211.0^{\circ}(4.9)$ & .59 \\
\hline $\begin{array}{l}\text { Rotated } \\
\text { card+entry }\end{array}$ & $\begin{array}{l}\text { 1-Card with Rotated } \\
\text { card and entry (B) }\end{array}$ & & 10 & $182.2^{\circ}(4.5)$ & .68 \\
\hline Rotated card & $\begin{array}{l}\text { 1-Card with Rotated } \\
\text { card }\end{array}$ & & 2 & $-5.5^{\circ}(5.5)$ & .68 \\
\hline Rotated entry & $\begin{array}{l}\text { 1-Card with Rotated } \\
\text { entry (B) }\end{array}$ & & 2 & $-6.0^{\circ}(2.0)$ & .76 \\
\hline
\end{tabular}

Rotational correlation analysis for cells in Category 1. Each row shows the mean over cells from the rotational correlational analysis (as described in Materials and Methods) between the firing-rate map for a standard session for each cell and those of the session type indicated for that row. In cases in which there was more than 1 session of a given type for a given cell, an analysis was conducted for each such session, and the average value over these sessions was used to calculate the mean over cells. Angle of $\operatorname{Max} R$ is the angle of rotation between the 2 maps at which a maximum $R$ value was obtained. Values close to 0 mean that fields for the session type in that row were in approximately the same angular location as in standard sessions. Max $R$ is the $R$ value at the Angle of Max $R$. High Max $R$ values mean that the fields were similar to standard sessions in size, shape, and distance from the cylinder center. Results for standard sessions indicate what these values are like when 2 standard sessions from the same cell are used for comparison. The numbers in parentheses are standard error values, and $N$ is the number of cells used for each comparison.

high chamber with cylindrical walls. The inner wall of the cylinder was uniformly gray, except for the presence of 1 or 2 movable, rectangular white cards, each of which covered $50^{\circ}$ of arc and extended the full height of the cylinder. The chamber had no floor but was laid on replaceable gray photo-backdrop paper. The entire cylinder was surrounded by a circular curtain $213 \mathrm{~cm}$ in diameter and $213 \mathrm{~cm}$ high. Illumination was provided by $415-\mathrm{W}$ light bulbs symmetrically arranged overhead.

Behavioral training. Prior to surgery, rats were trained in the cylinder to chase $20-\mathrm{mg}$ food pellets (BioServe, Frenchtown, NJ) that were thrown at approximately $15-\mathrm{sec}$ intervals to pseudorandom locations in the apparatus. During training, the cylinder was located in a separate room from the one in which cell screening and recording sessions were later conducted. During all training sessions, 1 white card was present on the cylinder wall. To begin each 16-min session, the rat was placed adjacent to the cylinder wall in a fixed relation to the card (this relation was identical to that for standard recording sessions; Table 1 and below). Daily training sessions were conducted for an approximately 2 -week period, during which the rats developed a pattern of nearly constant, apparently random locomotion that lasted throughout the sessions and resulted in them covering the entire floor of the apparatus fairly homogeneously.

Electrode implantation. After training, a drivable bundle of $10 \mathrm{mi}$ croelectrodes (Kubie, 1984) was chronically implanted in the brain. During surgery, the animals were anesthetized with a $40-\mathrm{mg} / \mathrm{kg}$ dose of pentobarbital, and supplemental doses were administered as necessary. The electrodes were constructed of formvar-insulated, $25-\mu \mathrm{m}$-diameter nichrome wire cut square at the ends and were implanted with their tips just above the CAl layer of the hippocampus (at skull coordinates of 4.5 posterior and 3.7 lateral to Bregma), in a position so that they could later be lowered through both the $\mathrm{CA} 1$ and $\mathrm{CA} 3$ cell layers of the dorsal hippocampus. Rats were allowed at least $10 \mathrm{~d}$ of recovery prior to the start of the experiment.

Unit isolation, data collection, and analysis. After recovery from surgery, the activity from each electrode was tested several times each day while the animal performed the pellet chasing task. The electrodes were gradually lowered until the activity from a single complex-spike cell (Ranck, 1973) could be isolated. 


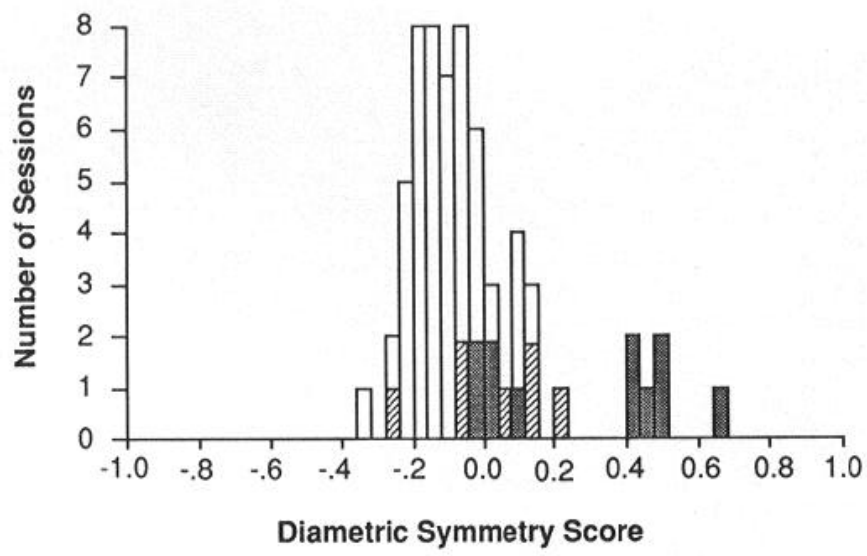

Figure 2. Histogram of diametric symmetry scores (see Materials and Methods) for each 2-card session for cells in Categories 1 and 2a. The shaded areas represent sessions from cells that were determined by visual assessment to have doubled their field on at least some sessions. There was a clearly bimodal distribution of scores, with all those in the second mode coming from cells that were visually assessed as having doubled on some sessions.

The signal from an active electrode was passed first through a fieldeffect transistor in source-follower configuration that was mounted in a rigid, lightweight headstage that interfaced with the connector on the animal's head. Signals then passed through a recording cable to a commutator that allowed the rats freedom of movement. The signal was amplified $(2000 \times)$ and filtered $(30 \mathrm{~Hz}$, high pass, and $10 \mathrm{KHz}$, low pass). The signal was then fed through a delay line and 2-time and amplitude-window discriminators that enabled isolation of waveforms coming from a single cell. Output pulses from the second discriminator were considered action potentials. The number of action potentials fired during each $1 / 60$-sec interval were counted and sent to a computer along with location information.

The animal's momentary location within the cylinder was also automatically recorded at a rate of $60 \mathrm{~Hz}$. For this, 2 small incandescent bulbs were attached to the rear of the animal's headstage. Signals from a video camera mounted above the cylinder were fed through a spot detector that detected the location of the headlights within a $256 \times 256$ grid of $2.9 \mathrm{~cm}^{2}$ pixels. (The camera field is the coordinate frame of all spatial representations presented below. All direction references such as " $3: 00$ " refer to angular coordinates as seen from the overhead camera.)

Each recording session lasted $16 \mathrm{~min}$. For each session, a firing rate map (see Figs. 3-7) for the isolated cell was constructed by dividing the total number of spikes that occurred when the animal was in each pixel by the total amount of time spent in each pixel. For visual presentation, the rates were color coded, with higher rates corresponding to darker colors, to produce a color map of firing rate over the area of the cylinder. Pixels that were not visited at all are shown in white, and yellow-coded pixels are those that were visited but in which the firing rate was exactly 0 .

In addition to visual inspection, these maps were assessed quantitatively. Two types of measures were used. First, it was necessary to compare spatial firing patterns between pairs of sessions so the effects of various stimulus manipulations could be evaluated. For this, a rotational cross-correlation was performed, in which the firing-rate map of one session was rotated through a series of $1^{\circ}$ shifts, and for each rotation a correlation between the rotated and the unrotated comparison map was made. This analysis provided 2 useful measures: (1) A determination of the angle at which the maximum correlation was obtained (Table 1, Angle of Max $R$ ) provided a measure of the firing-pattern rotation; (2) A determination of the magnitude of the correlation at this angle (Max $R$ ) provided a relative measure of the overall similarity of field shape, size, and distance from the center of the cylinder between 2 sessions. To obtain group averages for these correlation measures, each $R$ value was first transformed into a $Z$ score, and averages and standard errors for these values were obtained. The mean $Z$ score was then transformed back into an $R$ value. The second type of measure was a test of whether the firing pattern within any one session was diametrically symmetrical. For this, a correlation was performed be- tween a session's rate map and a $180^{\circ}$ rotation of that same map. The magnitude of the correlation is called the "diametric symmetry" score.

Testing paradigm. To begin a session, the animal was taken from its home cage, placed in an opaque cage, and carried into the circular curtain that enclosed the cylinder (see above). The cage was placed on the floor outside the cylinder near the designated entry location for that session. The animal was then picked up and held on the experimenter's shoulder while the recording headstage was attached. The animal was then placed into the cylinder, with its right side adjacent to the cylinder wall, in the designated starting location for that session. The carrying cage was immediately removed and the experimenter directly exited the curtained enclosure at the closest position. Recording then began, and pellets were thrown into the cylinder from varying locations around the outside of the curtained enclosure throughout the 16-min period. After completion of recording, the experimenter entered the enclosure in the same location exited from before, picked up the animal, detached the recording lead, replaced the rat into the carrying cage, and returned it to the home cage. There was an approximately $20-30$-min interval between sessions. A clean piece of gray floor paper was placed under the cylinder before the start of each session.

The first session in each series was a standard session (see Table 1). During standard sessions, a single cue card was located at 3:00 on the cylinder wall, while the animal's starting location was at 10:30. This configuration of cue card and entry location was identical to that used during training and electrode-screening sessions and enabled determination of the cell's field under familiar conditions.

For most cells (13 of 18), an additional 1-card session was conducted, identical to the first, except that both the cue card and entry position were rotated $180^{\circ}$ [Table 1 , rotated card + entry]. This enabled an assessment of whether the field location was determined largely by the card and entry position or by static, uncontrolled background cues.

Next, animals received a series of sessions in which a second card, identical to the first, was put on the wall $180^{\circ}$ from the first. The 2-card configuration created the ambiguous situation illustrated in Figure $1 B$, in which sets of identical locations were present $180^{\circ}$ apart and equidistant from the center. Several consecutive 2-card sessions were conducted for each cell. From one such session to the next, the entry position was alternated between the standard location (Table 1, 2-card-A), and a position $180^{\circ}$ from the standard (2-card-B). Thus, for these sessions, entry position was always the same angle relative to 1 of the 2 cards, but alternated between the 2 cards.

For some cells, modified 2-card session types were inserted into the series. In these, the 2 cards were each rotated together $30^{\circ}$ clockwise [Table 1, 2-card (+) nudge] or counterclockwise [2-card (-) nudge] from their usual locations. In addition, the entry position was shifted $180^{\circ}$ from the standard. These modified 2-card trials were run for 6 cells studied in later portions of the experiment, after it had been discovered that field position was controlled by entry location during unrotated 2-card sessions. This design dissociated entry position from its standard relation to either of the cue cards and thus tested the possibility that entry position was the only determinant of field location.

Following completion of 2-card sessions, an additional standard session was conducted. This tested whether field properties had changed over the day.

Finally, for 3 cells, 1 or 2 additional 1-card session types were conducted. These were designed to additionally test the relative importance of the entry position, the cue card, and the background cues in determining field location. In one type (Table 1, rotated card), the cue card was rotated $180^{\circ}$ degrees while entry position was held constant in relation to background cues. In the second type (rotated entry), entry position was rotated while the cue card was left in the standard position.

Histology. After all experimental recording animals were perfused transcardially under deep anesthesia with a formyl saline solution, their brains were sectioned at $40 \mu \mathrm{M}$ intervals and stained with Cresyl violet in order to determine electrode location.

\section{Results}

Recordings were made from a total of 18 cells in 12 animals. Nine were located in the CA1 hippocampal cell layer, and 9 were in CA3. No differences in the behavior of cells from these 2 regions could be detected, and this variable is not discussed further.

From inspection of color-coded rate maps, cells fell into 2 

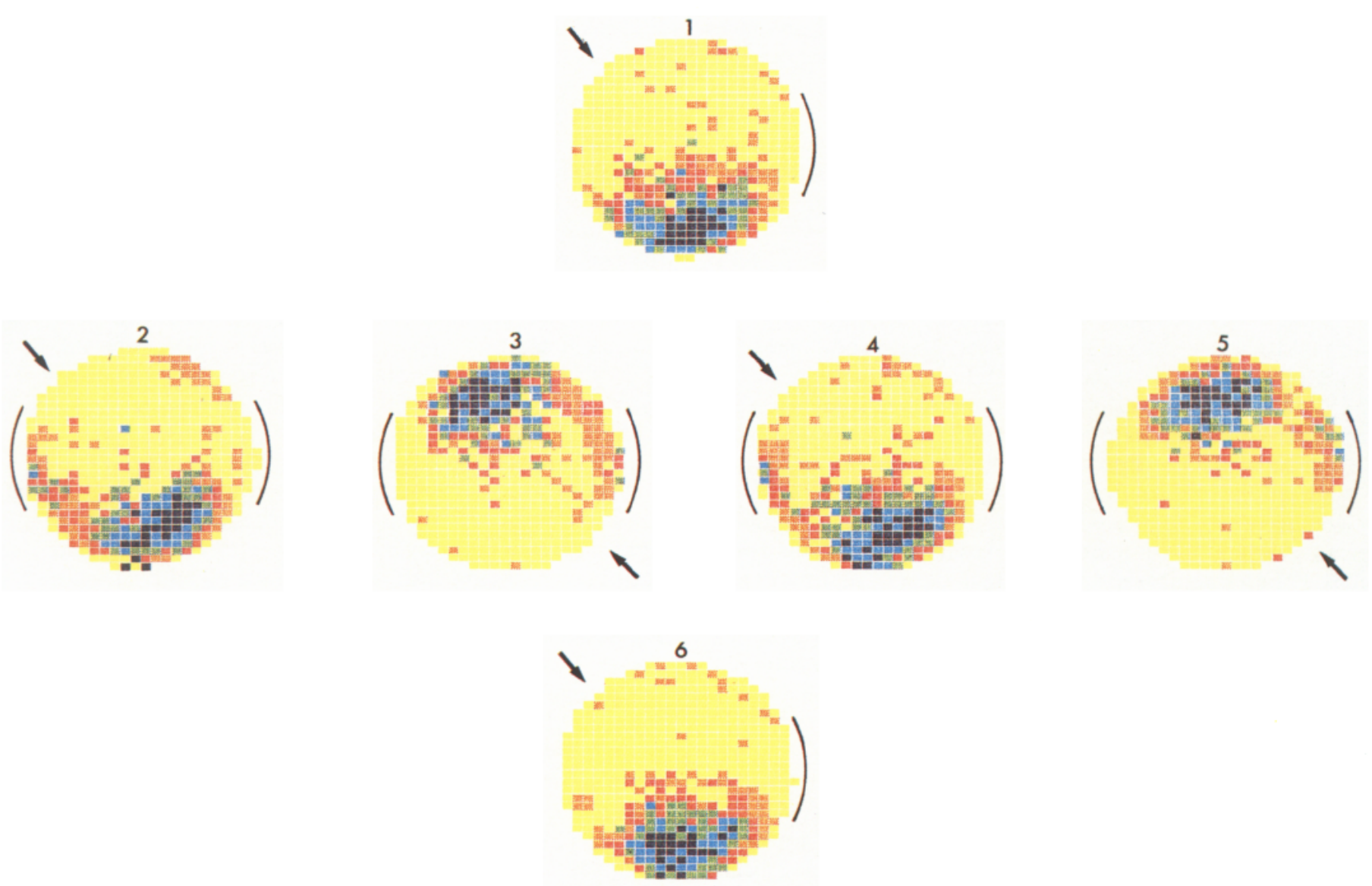

Figure 3. Firing-rate maps for cell in Category 1 over typical series of sessions. Each map shows the firing pattern over the area of the cylinder for a single 16-min session, with increasing rates coded in the following order: yellow, orange, red, green, blue, purple. Yellow pixels are those that the animal visited, but in which the cell never fired, and white pixels are those that were never visited. The absolute values of firing rate associated with each color are established on a relative basis. The arcs indicate the position of the cue card(s), and the arrows show the entry position for each session. Maps 1 and 6 were from standard sessions (see Table 1), with the card at 3:00 and the entry at 10:30. Maps 2-5 were from consecutive 2-card sessions with cards located at 3:00 and 9:00. Entry position was varied over these sessions between 10:30 and 4:30. It can be seen that the position of the firing pattern covaried with entry position for these latter sessions.

distinct categories with respect to their spatial firing patterns during the series of 2-cue sessions. This section is organized in terms of these response categories. Cells in Category 1 showed a single, asymmetric firing pattern similar to that in the standard situation throughout all sessions. Cells in Category 2 showed complex changes in firing patterns over the sessions. Category 2a is a subset of Category 2 including cells for which there was a clear doubling of firing fields in some sessions, producing spatial patterns with diametric symmetry. As a test of this qualitative categorization with regard to field doubling, all 2-card sessions for cells in Categories 1 and 2a were analyzed for diametric symmetry (see Materials and Methods). The results are plotted as a histogram in Figure 2, which shows a bimodal distribution of symmetry scores. All of the sessions from Category 1 cells (Fig. 2, open bars) fall in the lower mode, while some (but not all) of the sessions from Category 2a (darker bars) fall into the higher mode. Sessions for Category $2 \mathrm{a}$ cells that were judged by visual inspection to have symmetric firing patterns are shown in the darkest shading; sessions for the same cells that were considered to have asymmetric firing patterns are shown in lighter shading. With 1 exception, noted below, all cells in Category 2 came from different animals than those in Category 1.
Category 1: cells with a single, asymmetric firing pattern in all sessions

Most of the cells studied (13/18 in 8 animals) showed a single, characteristic spatial firing distribution in the presence of 1 or 2 cards. During both 1- and 2-card sessions, the firing patterns lacked diametric symmetry. The mean diametric symmetry score for these cells during 2-card sessions was $-0.11(Z=-0.11 \pm$ 0.02 ). Negative values are expected if the firing is asymmetric, because the firing rate at any spot is then systematically different from the rate across the diameter. During 2-card sessions, the field was of similar size and shape as during standard 1-card sessions and had the same position relative to 1 of the 2 cards that it had to the single card during standard sessions. In every cell but 1 , the card showing the standard relation to the field varied between sessions, so that on any one session, the field was at 1 of 2 possible positions, $180^{\circ}$ apart from one another. For these cells, the firing-field position was controlled by entry position; each time the entry position was rotated $180^{\circ}$, the field position rotated by $180^{\circ}$. The exceptional cell had a constant field location during all 2-card sessions that was identical to its position in standard sessions, indicating that entry position had no effect in this case. Figure 3 shows a typical cell during a series 
of 1 - and 2-card sessions. Map 1 shows the field in the presence of the standard stimulus configuration at the beginning of the recording day. The position of the white card is indicated by the curved line to the right of the firing-rate map. The entry position is indicated by an arrow. Maps 2-5 show successive 2-card sessions. For these sessions, the stimulus situation was constant, and only entry location was varied. Notice that the field location was invariably across the diameter from entry position and was therefore controlled by entry. For the 12 cells that showed control of firing pattern by entry location, a total of 46 2-cue sessions were conducted, and perfect control by entry location was observed in each case. Map 6 shows the field in the presence of the standard stimulus configuration, at the end of the 2-card series. The firing pattern was similar to that seen in the 1-card session at the beginning of the series (Map 1 ), indicating that the experience in the 2 -card environment had not altered the original firing pattern.

Rotation-correlation analyses (see Materials and Methods) were performed to compare probe sessions with standard sessions. For each comparison, the firing-pattern similarity is measured by the maximal correlation value among all angles (Max $R$ ). The amount of field rotation is taken as the angle at which the correlation was maximal (Angle of Max $R$ ). The rotationcorrelation analyses are shown in Table 1; values for the cell whose field did not vary with entry location are not included.

The first and last sessions of the test day are identical standard sessions with 1 cue card. When these sessions were compared with the rotational correlation method, the associated firing patterns were very similar. The mean rotation of the firing pattern was close to $0\left(2.7^{\circ}\right)$ and the $R$ scores were high (mean, $0.67 ; Z=0.70 \pm 0.07)$. Therefore, firing patterns were stable throughout the test day. Almost identical results were obtained when the initial session was compared with 2-cue sessions where the angle of entry was the same as standard sessions (Table 1, 2-card-A). Rotational analysis showed small rotations of the firing pattern (mean rotation, $-2.3^{\circ}$ ) and high $R$ scores (mean, $0.72 ; Z=0.91 \pm 0.09$ ). This outcome supports the idea that firing patterns during 2-card-A sessions are indistinguishable from replications of the standard session. When the initial standard session was compared with 2-card sessions in which the angle of entry was rotated $180^{\circ}$ (2-card-B), a high mean $R$ was again found, indicating that the shape and radial position of the firing fields were unchanged (mean $R, 0.60 ; Z=0.70 \pm 0.07$ ). As expected if entry position controls the angular position of fields, the patterns rotated approximately $180^{\circ}$ (mean, $182^{\circ}$ ).

The results shown so far are compatible with the idea that entry location is the only salient cue for firing field position; it is sufficient by itself to predict field location. Probes were therefore conducted to test whether the cards also exerted control. In one type of probe (Table 1, 2-card-nudge), 2-card sessions were conducted with the cards rotated $30^{\circ}$ clockwise or counterclockwise from their usual positions, with entry location $180^{\circ}$ away from that in the standard sessions. If angle of entry has complete control over firing patterns, the fields should be $180^{\circ}$ away from their position in the standard configuration. Alternatively, if the cards are salient, the fields should rotate $30^{\circ}$ in the same direction as the cards.

The results show that the fields rotate approximately $30^{\circ}$ in the same direction as the cards, demonstrating the salience of the cue cards. Figure 4 shows a typical cell during a series of sessions including $30^{\circ}$ rotations. Maps 2, 3, 5, and 6 show 2-card sessions with the cards at 3:00 and 9:00. During these sessions, the firing field either is in its standard position or has rotated $180^{\circ}$, depending on entry location. Maps 4 and 7 show the field when the cards are rotated $30^{\circ}$ clockwise (Session 4 ) or counterclockwise (Session 7), with entry location kept the same as in Sessions 3 and 6 . The field rotates in the same direction as the cards during each of Sessions 4 and 7 . Notc also that entry location is of predictive value even in these sessions, because field location was closest to that predicted on the basis of the $180^{\circ}$ rotated entry position, rather than the standard entry position. Over all cells tested, clockwise rotations produced an average maximum angle of $158.3 \pm 5.5^{\circ}$ (about $22^{\circ}$ less than that predicted by entry location alone), with $R$ values of 0.65 $(Z=0.78 \pm 0.09)$. Counterclockwise rolations resulted in angular values of $211.0 \pm 4.9^{\circ}(R=0.59 ; Z=0.68 \pm 0.06)$, which is $31^{\circ}$ more than that predicted by entry location alone.

Further tests of the influence of the cue card and entry position on field location were conducted in 2 cells using 1 -card sessions. These involved $180^{\circ}$ rotation of either the card alone (Table 1 , rotated card) or the entry position alone (rotated entry) and were used in conjunction with sessions in which both cues were rotated together (rotated card + entry) to determine the relative importance of the card, entry position, and background cues. Figure 5 shows the results for the 3 session types for the same cell as in Figure 4. When both card and entry location were rotated (Map 2), the field also rotated $180^{\circ}$ from its location during standard sessions (Map 1). When only entry location (Map 3) or only the card (Map 4) were rotated, however, the field remained in the standard location. Therefore, if the total stimulus configuration is taken to consist of 3 separate cues (cue card, entry location, and static background cues), the firing field location was always controlled by the pattern of cues that went together. Only 2 cells were tested under these conditions; both showed this pattern, and Table 1 shows fring pattern rotation values and $R$ values for each of these session types.

\section{Category 2a: cells with a fing pattern that changed over time and was symmetrical for some sessions}

Three of the 18 cells (in 3 rats) showed a pattern that changed over sessions and showed paired, symmetrical fields on at least some sessions. With one exception (see below), these cells were obtained from animals different from the animals that yielded Category 1 cells. Cells in this category were characterized by variability in their firing patterns over trials, with activity differing from one session to the next, even under identical conditions. These cells showed more than 1 field for at least some of the 2-card sessions, such that the pattern was a doubling of the original, standard field, with the second field located $180^{\circ}$ away from the first. The mean diametric symmetry value for those sessions that, by visual examination, appeared to have doubled fields was $0.39(Z=0.41 \pm 0.03)$. This indicates a high degree of diametric symmetry and is in sharp contrast to the mean of -0.11 for such sessions in Category 1 cells. One cell in this category virtually ceased firing during some sessions.

The spatial firing patterns of the first Category $2 \mathrm{a}$ cell are shown in Figure 6 . On the first of 2 recording days, the cell showed a dramatic reduction in firing rate during the first 2 sessions with 2 cards; firing was so weak that no field could be discerned. During the next 2 sessions ( 4 and 5 ), the cell recovered its initial rate and showed a single field, the location of which was predicted by entry location. On day 2 , however, the cell showed a doubled field during all 2-card sessions. Interestingly, this doubled field remained even during the subsequent 2-card 

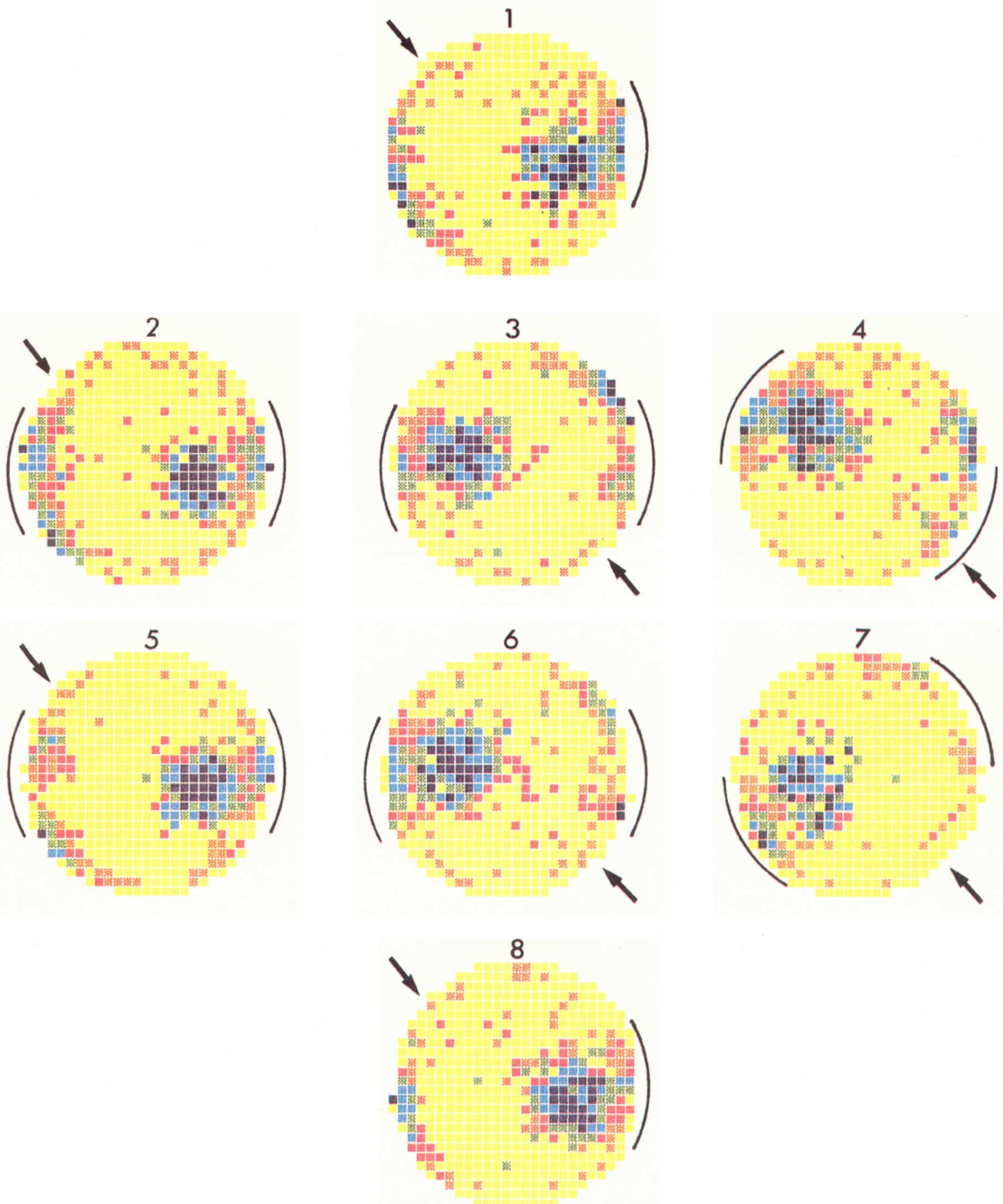

Figure 4. Firing-rate maps for different cell (in a different animal) in Category 1. Maps 1 and 8 were from standard sessions. Maps 2-7 show a series of 2-card sessions. In Sessions 2, 3, 5, and 6, the cards were at 3:00 and 9:00, and entry position was varied between 10:30 and 4:30. The firing pattern covaried with the entry position during these sessions. In Sessions 4 and 7, the cards were both rotated $30^{\circ}$ clockwise (Session 4 ) or counterclockwise (Session 7), while the entry position was at 4:30. In these cases, the firing pattern also rotated $30^{\circ}$ (in the same direction as the cards) from its usual position when the entry position was $4: 30$. The arcs indicate the position of the cue card(s), and the arrows show the entry position for each session. Colors are as for Figure 3. 

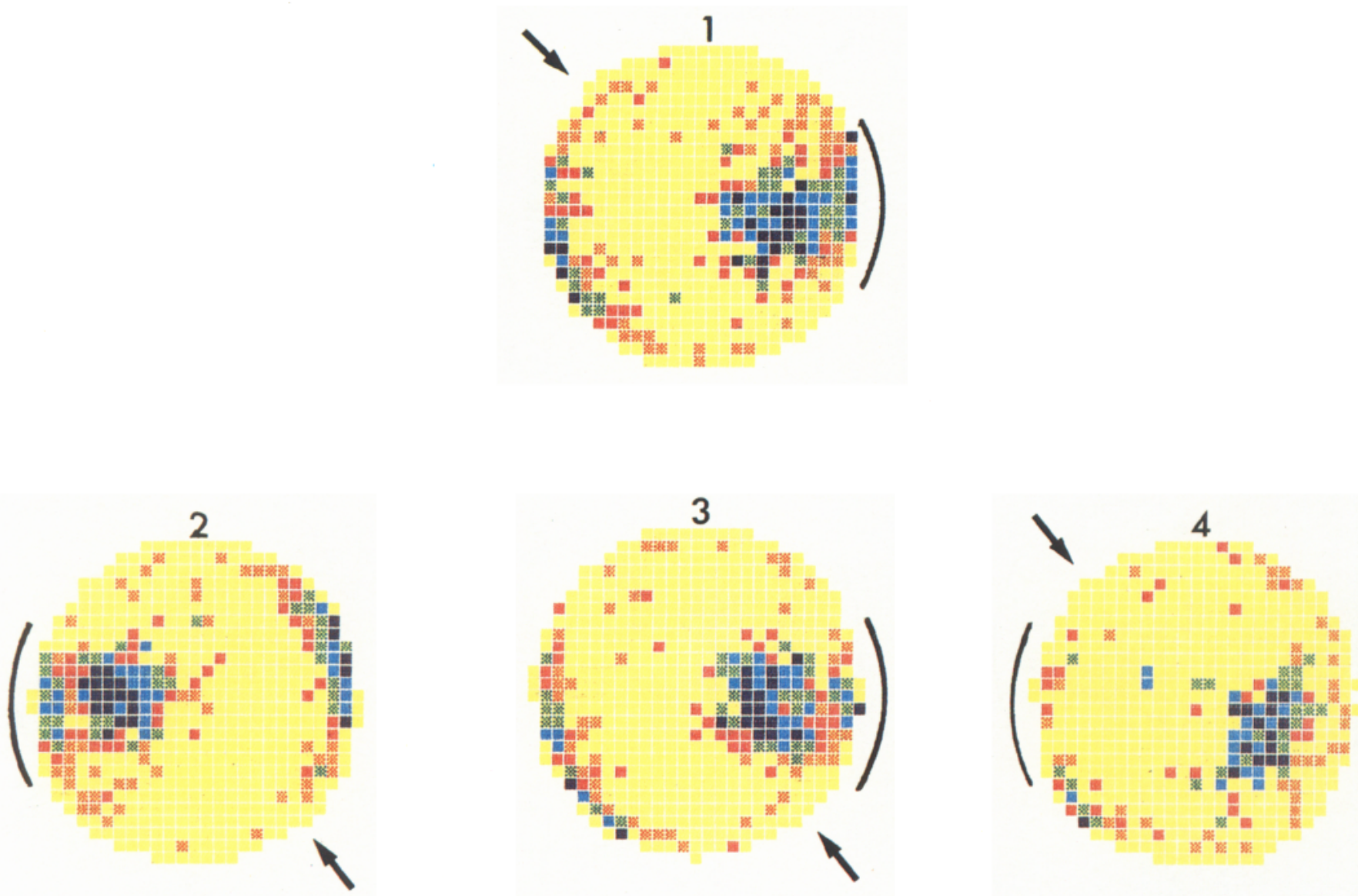

Figure 5. Firing-rate maps for same cell shown in Figure 4. Map 1 illustrates a standard session while Maps 2-4 are from a series of 1-card sessions designed to test the relative salience of the card, entry position, and background cues. In Session 2, the card and entry position were both rotated $180^{\circ}$ from the standard. In Session 3, only the entry position was rotated, while in Session 4, only the card was rotated. In these latter 3 sessions, firing patterns were located in the position in which they had the same relationship as in the standard session to as many of the cues as possible. The arcs indicate the position of the cue card, and the arrows show the entry position for each session. Colors are as for Figure 3.

session. Therefore, the doubling of the field caused by repeated presentations of the 2-card environment was retained when the second card was no longer present. An additional 1-card session run $30 \mathrm{~min}$ after Session 12 in Figure 6 also exhibited the doubled pattern. This unexpected result is again different from that predicted from the simple sensory model.

Figure 7 shows a second cell with an unstable firing field recorded from a different animal. This cell was also recorded over a 2-day period. It showed a doubled field during the first 2 -cue session (but not during any 1 -card session). Over repeated 2 -card sessions, however, 1 of the 2 fields progressively weakened, so that the doubled pattern gradually transformed into a single-field pattern. Again, this is not compatible with the simple sensory model. The single field rotated in a manner predicted by entry location. In the same animal, a second cell was sub- sequently isolated for recording. This cell showed a single, asymmetric field throughout all sessions and was included in the results for Category 1 cells above.

The third cell in this category was held only long enough to record the standard 1-card session and 12 -card session. This cell showed a doubled field in the presence of the 2 cards.

Category 2b: cells with a firing pattern that changed over time but did not show symmetrical firing patterns

The first cell in this class was a second unit from the same animal as the third cell in Category 2a. This cell showed complicated changes over sessions, including changes in the shape and number of firing fields. The second cell in Category $2 b$ showed a pattern consisting of 3 stages: (1) three 2-card sessions during which there was a single field of the same size, shape, and lo-

Figure 6. Firing-rate maps from cell in Category 2. Day 1, Maps 1 and 6 are from standard sessions. Maps 2-5 show a series of 2-card sessions with the cards at 3:00 and 9:00 and in which entry position was varied between 10:30 and 4:30. The cell was virtually silent during Sessions 2 and 3. Firing reappeared during Sessions 4 and 5, and covaried with entry position. Day 2, Maps 7 and 12 are from standard sessions. Maps 8-11 are from a series of 2-card sessions as in Day 1. Here, the firing field doubled, so that there were 2 fields $180^{\circ}$ apart and equidistant from the center during all 2-card sessions. Interestingly, this doubled pattern remained even during Session 12, when only 1 card was present. The arcs indicate the position of the cue card(s), and the arrows show the entry position for each session. Colors are as for Figure 3. 
DAY 1

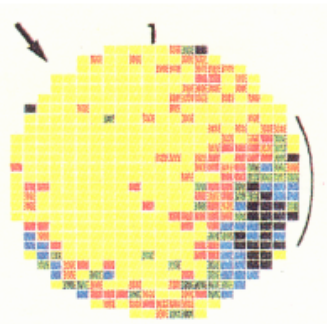

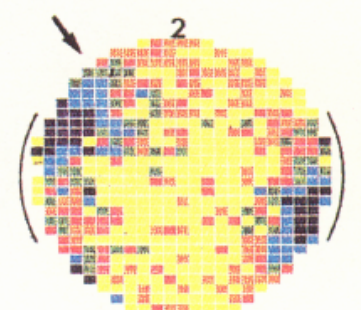

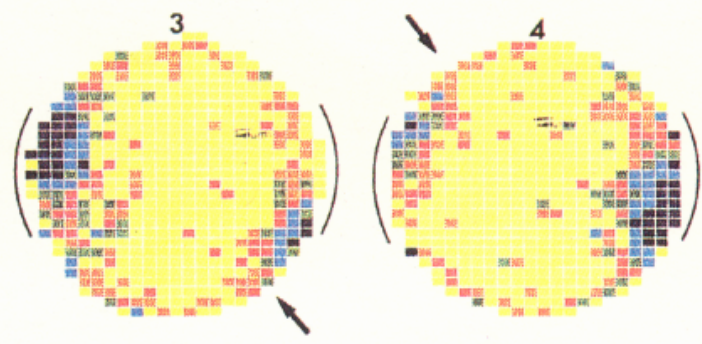

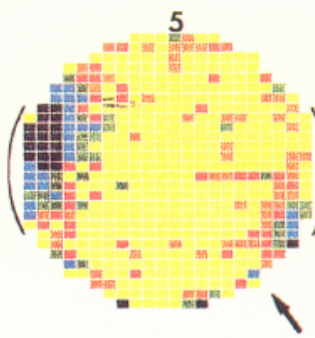

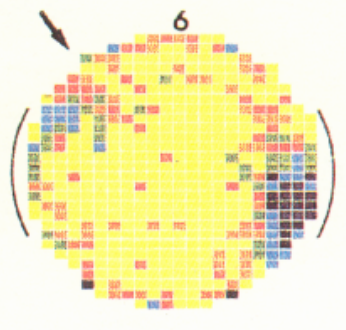

DAY 2
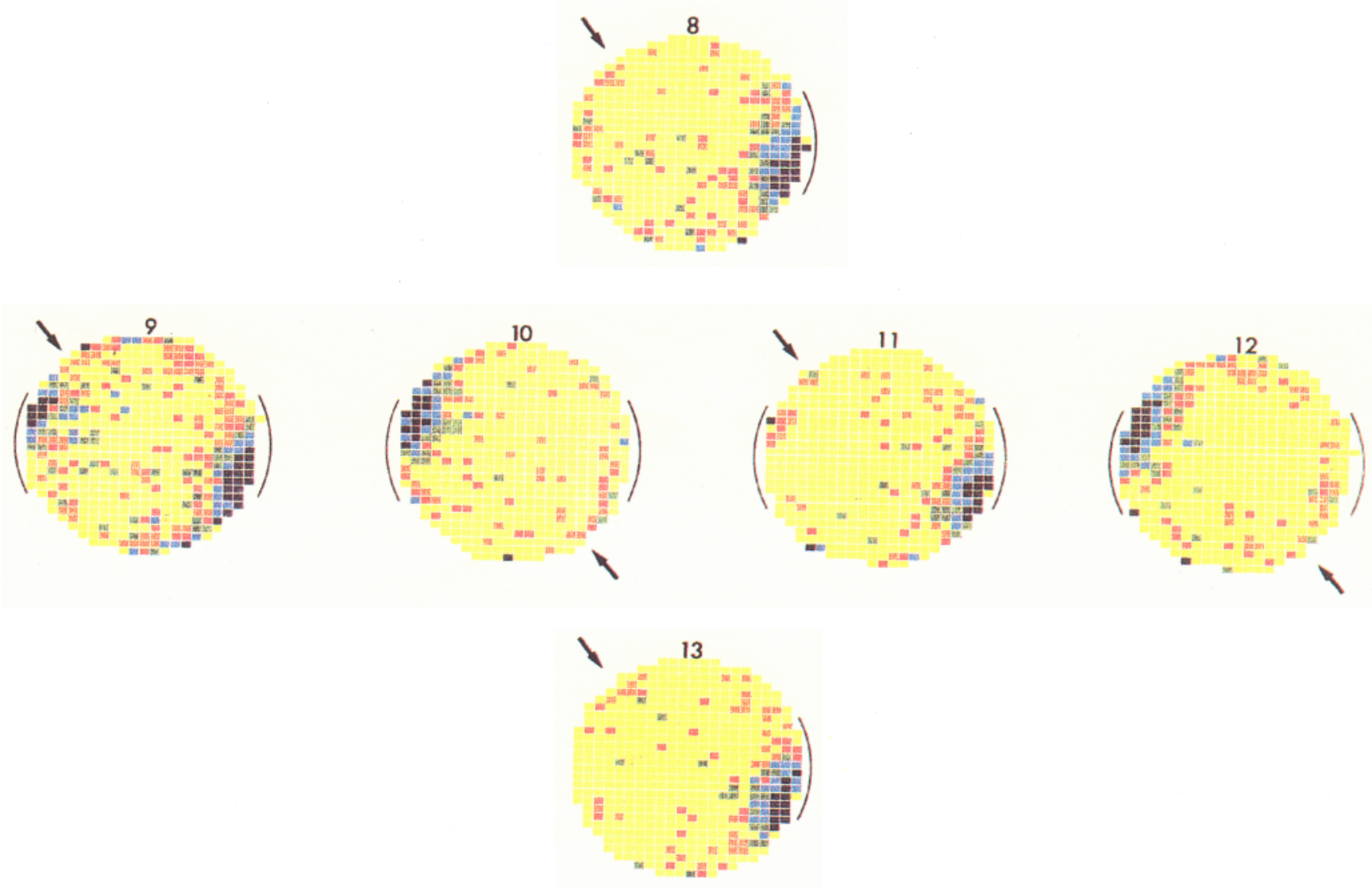

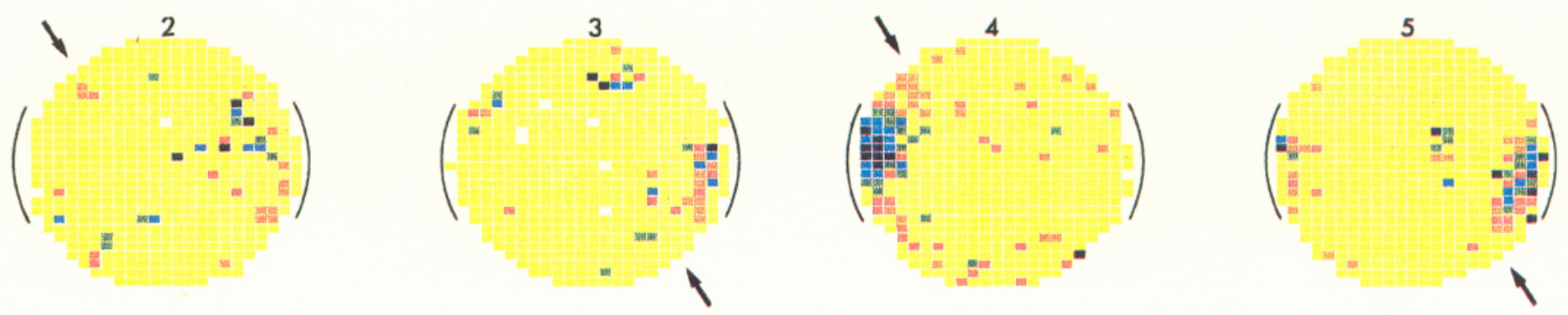

DAY 2
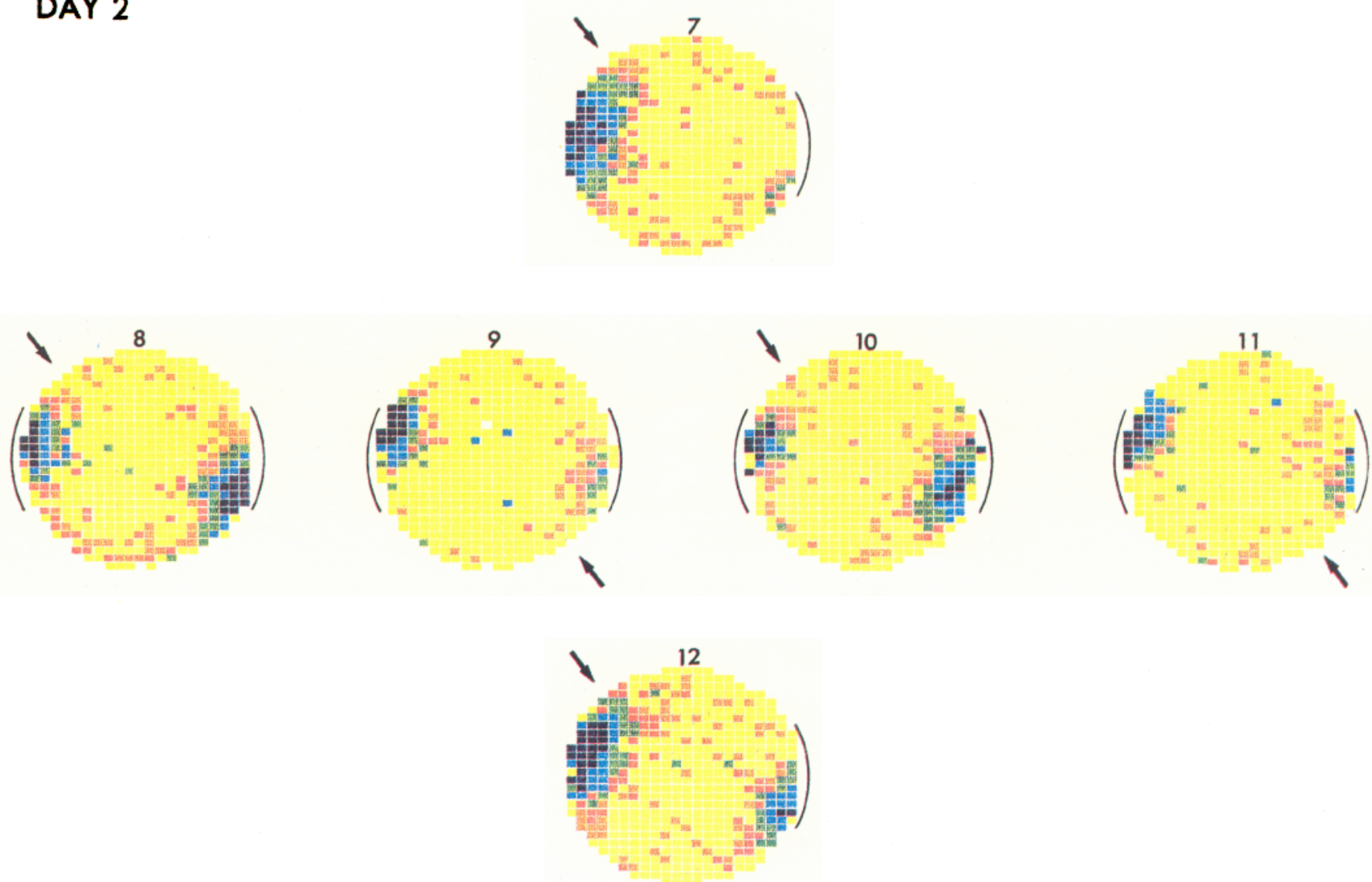
cation as in the standard environment; during these sessions the field did not rotate along with entry location; (2) two 2-card sessions during which there was almost no firing; and (3) 3 additional 2-card sessions during which the single field reappeared and rotated in relation to entry location.

\section{Discussion}

The majority of cells tested in the symmetric, 2-card situation showed differential firing rates in visually similar locations $180^{\circ}$ apart. In all cases, for cells of this type, the fields were approximately the same size, shape, and distance from the center of the cylinder as during the standard 1-card sessions. Also, they had the same relationship to 1 of the 2 cards as they had to the single card during standard sessions. In every case but 1 , the particular card showing this standard relationship to the field varied between sessions but was constant within any one session. The particular card showing a standard relationship to the firing field on any particular 2-card session did not vary randomly but could be selected by rotations of the animal's entry location. When entry was varied between the standard and the diametrically opposite location, the field varied in such a way as to maintain the standard relationship to entry.

These results provide strong evidence that the simple sensory model of hippocampal cell firing is not adequate, by itself, to explain the cell firing observed here. Rather, mnemonic factors also play a role. This is true because pairs of locations that were designed to be visually identical were represented in very different ways by the hippocampus over the course of any one session, and the orientation of this asymmetrical pattern was determined by the transient entry-location cue. The fact that entry location is a transient cue means that its influence on the firing pattern over the course of each 16-min session must have been based on mnemonic processes.

There are 2 important caveats that must be made in relation to the above conclusions: (1) Although the 2-cue environment was designed to provide perfect diametric symmetry, it may be that uncontrolled factors, such as noise from the equipment or unintentional differences between the cards, have made pairs of nominally identical locations somewhat different in the sensory input they provide. Although this possibility cannot be ruled out, the mnemonic interpretation is strengthened by the fact that the fields alternated between 2 nominally identical locations across different sessions, thus demonstrating that the 2 locations could be treated equivalently by the hippocampal system. The fact that the 2 locations were not treated identically within any one session suggests that a mnemonic process must have been involved in the differentiation. (2) It is possible that the entry location was not a transient cue but that some residual sensory trace, such as an odor, was left behind by the original entry event. This seems unlikely because sessions were run in fairly rapid succession, which might be expected to be produce interference between the 2 possible entry locations from session to session. Also, no obvious form of marking (e.g., urination) was observed at entry.

These results are further confirmation of the importance of memory for spatial firing as reported by O'Keefe and Speakman (1987), Quirk et al. (1990), and Jones-Leonard et al. (1985). In these studies, extinguishing the lights or removing salient cues did not affect cell firing. Our work shows that, in addition, a cue that is only transiently present can control field location, even in the continued presence of the demonstrably salient cue cards.

The partial dependence of firing patterns on a trace cue is generally compatible with lesion studies that indicate hippocampal involvement in working (Olton et al., 1979) or intermediate-term (Rawlins, 1985) memory. The ability of the hippocampus to maintain a consistent differentiation between a pair of locations may be a reflection of such memory functions, where the memory resides either in the hippocampus or elsewhere in the brain. Support for the idea that the hippocampus itself is responsible for this type of memory function has been provided by recent data in monkeys, where it was found that the hippocampus is preferentially active during working memory tasks (Friedman and Goldman-Rakic, 1988). Also, evoked potential amplitude in the hippocampal formation is affected by events occurring over several minutes prior to the occurrence of the evoking stimulus (West et al., 1982; Sharp et al., 1989).

The covariation of firing field location with entry position during 2-card sessions suggested the possibility that, in this situation, entry location was the only relevant cue for determining field location, and that the cue cards had lost their salience because they no longer provided adequate polarizing information. However, probes designed to test the influence of the cue cards on cell firing showed that this was not the case. During 2 -card sessions in which the pair of cards was rotated $30^{\circ}$ while entry location was held constant, fields rotated in register with the cards, demonstrating that the cards were salient. Interestingly, of the 2 possible visually identical locations, fields were located in the one closest to that of the standard in relation to angle of entry. Therefore, both the cards and the entry location had an influence on field location. Although in a single session a cell is influenced by both entry and cue factors, it is not responding to them as independent influences that simply add together. Rather, the cards and entry location are combined in such a way that the otherwise unchanged field rotates to a specific angular position; the fields do not stretch or rotate by an intermediate value. Specifically, the gross angular position of firing fields is determined by entry position, and fine resolution of angular position is determined by the cards.

Further probes using variations of the 1-card situation showed that, in addition to the cue cards and the entry location, uncontrolled background stimuli can also play a role. In sessions in which both the card and entry location were rotated $180^{\circ}$ relative to the background, the fields also rotated $180^{\circ}$. However,

$\leftarrow$

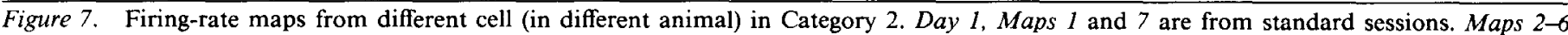

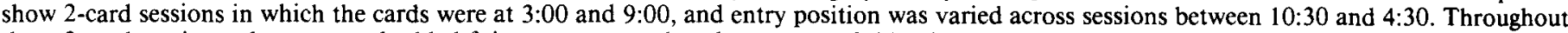

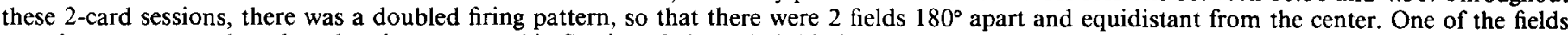

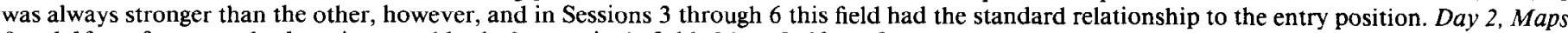

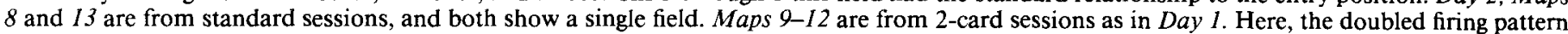

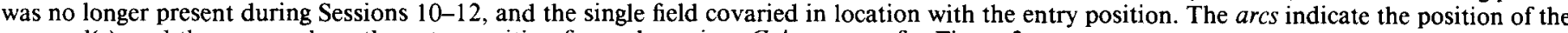
cue card(s), and the arrows show the entry position for each session. Colors are as for Figure 3. 
when only 1 of these 2 cues was rotated, the cells fired in the standard location, so that they were in the standard relationship to both the background cues and the unrotated cue (card or entry location). Therefore, the 2 cells tested always fired in such a way that they were in the standard relationship to 2 of 3 possible cues (i.e., background, cue cards, and entry location).

It should be noted that the results for the cue-card-alone rotations in this study are different from those found by Muller and Kubie (1987), which showed control of firing fields by card rotations. The most likely reason for this difference is that, in the latter study, entry location was not necessarily held constant across sessions as it was here.

In the results discussed so far, fields in each type of probe session were of similar size, shape, and distance from the cylinder center as in standard sessions. The location of any single field was influenced by several cues and seemed to result in the field having the same relationship as in the standard sessions to as many of the cues as possible. This pattern is in contradistinction to other possible results. For example, it would have been possible to see multiple fields, each having the same relation to one of the cues as in the standard; the rotation of the field to an intermediate position; the stretching of fields; or the appearance of a completely new field, unpredictable in size, shape, and location from that in the standard. The pattern of results suggests that place cells use a mnemonic process to encode the stimulus configuration in their field in the original training situation and then fire in the place that most closely resembles the original field.

These results are compatible with the idea that the hippocampus is involved in configural learning (Hirsh, 1974; Sutherland and Rudy, 1989). According to this position, the hippocampus is responsible for learning on tasks in which the relevant stimuli are configurations of separable stimuli, rather than individual stimuli. Here, the place-cell firing itself is viewed as a learned response to the configuration of stimuli available to an animal when it is in a particular place. It can be reinstated on subsequent occurrences of these configurations (places) and can do so even when the environment has becn modified somewhat.

Category 2 cells produced patterns of response not easily reconcilable with simple predictions. These cells showed variations in their firing patterns over 2-card sessions and, in 1 case, showed an altered pattern in 1 -card sessions after the 2-card exposures. Thus, these cells showed plasticity in their firing pattern within an environment as a result of a change in that environment. In this way, our results are similar to those of Bostock et al. (1986), who also showed a change over time in hippocampal firing patterns over repeated presentations of a novel-stimulus situation.

Cells in Category 2 a showed field doubling (diametric symmetry). The fact that these cells sometimes showed such paired fields suggests that, at least for these cells, there is some tendency to exhibit identical firing patterns in visually identical locations. Thereforc, local stimulus configuration may have a strong influence. However, even for these cells, the firing cannot be explained in terms of triggering by the local stimulus configuration because symmetric fields were present during only some of the sessions and could persist when the second card was removed.

Although the firing patterns over the entire set of 2-card sessions showed considerable variability for Category 2 cells, there was usually a relative stability between any 2 consecutive 2-card sessions. For instance, the last few 2-card sessions were generally similar in pattern, suggesting that the system had reached a new steady state by that time. This kind of local temporal stability suggests that changes in the system resulting from 2-card exposures last at least over a period of a few sessions; that is, variables in the system that are responsible for the particular firing pattern exhibitcd on trial $N$ arc stable over the intertrial interval, so that firing on trial $N+1$ is relatively similar. Therefore, relatively long-lasting changes in the system may play a role in determining firing patterns. This notion fits the fact that the hippocampus is involved in learning on spatial tasks (O'Keefe and Nadel, 1978; Olton et al., 1978; Morris et al., 1982, 1986; Jarrard, 1983; Sutherland et al., 1983) and suggests that the representation of the environment provided by the pattern of hippocampal pyramidal cell firing is a learned representation based on past experience in the environment. This point is made particularly clearly by the cell that retained a doubled field even when the second card was removed (see Fig. 6). The firing pattern exhibited during this session must have been based on past experience in the 2 -card version of the environment.

In summary, these results have extended previous observations that hippocampal place cells do not respond simply on the basis of immediately available sensory cues. Rather, there is evidence that both long-lasting and short-lasting mnemonic processes are involved. The role of short-lasting mnemonic processes is suggested by the fact that the field location was influenced throughout any one 16-min session by the session-specific, transient entry location cue. Long-lasting processes were demonstrated by the fact that introduction of a second cue into the environment caused, in some cells, gradual changes in the overall firing pattern in that environment, and these changes extended over time across sessions. These results are compatible with others showing both short- and long-lasting environmentally induced changes in evoked potentials in the hippocampal formation (Ruthrich et al., 1982; Weisz, 1982; Sharp et al., 1985, 1987, 1989; Skelton et al., 1987), as well as the well-known electrically induced plasticity (long-term potentiation) that has becn discovered in these responses (Bliss and Gardncr-Medwin, 1973; Bliss and Lomo, 1973). These results allow for the possibility that the neuronal plasticity reflected in these evoked potential changes may be responsible for the recoding of environmental information in individual pyramidal cells observed here and in an earlier study (Bostock et al., 1986) in response to environmental changes.

\section{References}

Bliss TVP, Gardner-Medwin AR (1973) Long-lasting potentiation of synaptic transmission in the dentate area of the unanesthetized rabbit following stimulation of the perforant path. J Physiol (Lond) 232: 357-374.

Bliss TVP, Lomo T (1973) Long-lasting potentiation of synaptic transmission in the dentate area of the anaesthetized rabbit following stimulation of the perforant path. J Physiol (Lond) 232:331-356.

Bostock E, Muller RU, Kubie JL (1986) Firing fields of hippocampal neurons: a stimulus manipulation that alters place cell mapping of the environment. Soc Neurosci Abstr 12:522.

Friedman HR, Goldman-Rakic PS (1988) Activation of the hippocampus and dentate gyrus by working memory: a 2-dcoxyglucosc study of behaving rhesus monkeys. J Neurosci 8:4693-4706.

Hirsh R (1974) The hippocampus and contextual retrieval of information from memory: a theory. Behav Biol 12:421-444.

Jarrard LE (1983) Selective hippocampal lesions and behavior: effects of kainic acid lesions on performance of place and cue tasks. Behav Neurosci 97:873-889.

Jones-Leonard B, McNaughton BL, Barnes CA (1985) Long-term 
studies of place field interrelationships in dentate gyrus neurons. Soc Neurosci Abstr 11:1108.

Kubie JL (1984) A driveable bundle of microwires for collecting single unit data from frccly moving rats. Physiol Behav 32:115-118.

McNaughton BL (1989) Neuronal mechanisms for spatial computation and information storage. In: Neural connections, mental computation (Nadel L, Cooper LA, Culicover P, Harnish RM, eds), pp 285-349. Cambridge, MA: MIT.

McNaughton BL, Leonard B, Chen L (1989) Cortical-hippocampal interactions and cognitive mapping: a hypothesis based on reintegration of the parietal and inferotemporal pathways for visual processing. Psychobiology 17:236-246.

Morris RGM, Garrud P, Rawlins JNP, O’Keefe J (1982) Place navigation impaired in rats with hippocampal lesions. Nature 297:681683.

Morris RGM, Hagan JJ, Rawlins JNP (1986) Allocentric spatial learning by hippocampectomized rats: a further test of the "spatial mapping" and "working memory" theorics of hippocampal function. Q J Exp Psychol 38B:365-395.

Muller RU, Kubie JL (1987) The effects of changes in the environment on the spatial firing of hippocampal complex-spike cells. J Neurosci 7:1951-1968.

Muller RU, Kubie JL, Ranck JB Jr (1987) Spatial firing patterns of hippocampal complex-spike cells in a fixed environment. J Neurosci $7: 1935-1950$

O'Keefe J (1976) Place units in the hippocampus of the freely moving rat. Exp Neurol 51:78-109.

O'Keefe J, Conway DH (1978) Hippocampal place units in the freely moving rat: why they fire where they fire. Exp Brain Res 31:573-590

O'Keefe J, Dostrovsky J (1971) The hippocampus as a spatial map: preliminary evidence from unit activity in the freely moving rat. Brain Res 34:171-175.

O'Keefe J, Nadel L (1978) The hippocampus as a cognitive map. New York: Oxford UP.

O'Keefe J, Speakman A (1987) Single unit activity in the rat hippocampus during a spatial memory task. Exp Brain Res 68:1-27.

Olton DS, Walker JW, Gage FH (1978) Hippocampal connections and spatial discrimination. Brain Res 139:295-308.

Olton DS, Becker JT, Handelmann GE (1979) Hippocampus, space and memory. Behav Brain Sci 2:352-359.
Quirk G.I, Muller RU, Kubie JL (1990) The firing of hippocampal place cells in the dark depends on the rat's recent experience. J Neurosci 10:2008-2017.

Ranck JB Jr (1973) Studies of single neurons in dorsal hippocampal formation and septum in unrestrained rats. Exp Neurol 41:461-555.

Rawlins JNP (1985) Associations across time: the hippocampus as a working memory store. Behav Brain Sci 8:479-496.

Ruthrich H, Matties H, Ott T (1982) Long-term changes in synaptic excitability of hippocampal cell populations as a result of training. In: Neuronal plasticity and memory formation (Marsan CA, Matties H, eds), pp 589-594. New York: Raven.

Sharp PE (1989) Computer simulation of hippocampal place cells. Soc Neurosci Abstr 15:403.

Sharp PE, McNaughton BL, Barnes CA (1985) Enhancement of hippocampal field potentials in rats exposed to a novel, complex environment. Brain Res 339:361-365.

Sharp PE, Barnes CA, McNaughton BL (1987) Effects of aging on environmental modulation of hippocampal evoked responses. Behav Neurosci 101:170-187.

Sharp PE, McNaughton BL, Barnes CA (1989) Exploration-dependent modulation of evoked responses in fascia dentata: fundamental observations and time course. Psychobiology 17:257-269.

Skelton RW, Scarth AS, Wilkie DM, Miller JJ, Philips AG (1987) Long-term increases in dentate granule cell responsivity accompany operant conditioning. J Neurosci 7:3081-3087.

Sutherland RJ, Rudy JW (1989) Configural association theory: the role of the hippocampal formation in learning, memory, and amnesia. Psychobiology 17:129-144.

Sutherland RJ, Wishaw IQ, Kolb B (1983) A behavioral analysis of spatial localization following electrolytic, kainate- or colchicine-induced damage. Behav Brain Res 7:133-153.

Weisz DJ (1982) Activity of the dentate gyrus during nictitating membrane conditioning in the rabbit. In: Conditioning: advances in behavioral biology, Vol. 26, representations of involved neural functions (Woody CD, ed), pp 131-146. New York: Plenum.

West MO, Christian E, Robinson JH, Deadwyler SA (1982) Evoked potentials in the dentate gyrus reflect the retention of past sensory events. Neurosci Lett 28:319-324.

Zipser D (1985) A computational model of hippocampal place fields. Behav Neurosci 99:1006-1018. 